\title{
Beiträge zur vergleichenden Pflanzenchemie
}

\section{II. Über die Beerenfrüchte einiger Caprifoliaceen}

\author{
Von \\ Gisela Nowak und Julius Zellner
}

(Vorgelegt in der Sitzung am 14. Juli 1921)

Die folgenden Ausführungen beziehen sich auf die vergleichendchemische Untersuchung einer Reihe von Caprifoliaceenfrüchten. Die Früchte dieser Gruppe sind noch wenig untersucht, die einschlägige Literatur wird bei den einzelnen Arten angeführt werden. Die untersuchten Materialien waren von uns gesammelt worden mit Ausnahme der Früchte von Sambucus nigra, die von der Firma Petzold \& Süß gekauft und von Kustos Rechinger identifiziert wurden. Aus äußeren Gründen mußten die Früchte in lufttrockenem Zustand und samt den Samen untersucht werden, zwei Umstände, die für pflanzenchemische Untersuchungen nicht gerade günstig sind.

Um Wiederholungen $z u$ vermeiden, sei bemerkt, daß in allen Fällen zunächst eine qualitative Untersucnung der Früchte vorgenommen wurde, an die sich dann eine quantitative Bestimmung der wichtigeren Bestandteile anschloß. In den meisten Fällen wurde auch das Verhältnis zwischen Beeren- und Samengewicht festgestellt, weil dadurch Schlüsse auf die Zusammensetzung des Fruchtfleisches ermöglicht sind.

\section{Lonicera Xylosteum L.}

Rote Doppelbeeren von fad süßlichem Geschmack. Das Material wurde in Mürzzuschlag (Steiermark) gesammelt.

Über dié chemische Zusammensetzung liegen einige, vorwiegend ältere Angaben ${ }^{1}$ vor, die durch unsere Untersuchung bestätigt wurden.

1 Hübschmann, Viert.-Jahrschr. f. prakt. Pharm., 1845/197; Enz, Viert.Jahrschr. f. prakt. Pharm., 1856/196; Wo1ff, Aschenanalysen, I/127; Bridel, Journ. de Pharm. et Chimie, $1907 / 536$. 
Die qualitative Untersuchung des Fruchtfleisches ergab folgendes:

im Petrolätherauszug findet sich ein fettes Öl, das nur in sehr geringer Menge vorhanden ist und daher nicht näher untersucht wurde;

im Atherauszug wachsartige Stoffe neben einem intensiv gelb gefürtbten Körper, der in Fettlösungsmitteln löslich ist (Lipochrom). Schimper ${ }^{1}$ fand auf mikroskopischem Wege rosenrote und orangefarbige Krystalle von starkem Pleochroismus, die er als Carotene anspricht. Außerdem läßt sich aus dem Rückstand der Ätherextraktion mit heißem Wasser das Xylostein gewinnen, das zuerst Hübschmann (l. c.) isoliert hat. Leider hatten wir nur getrocknetes Material zur Verfügung, aus dem infolge einer beim Trocknen erfolgenden Säurehydrolyse oder Fermentwirkung weder Xylostein noch ein krystallisierbarer Abkömmling desselben isoliert werden konnte.

Eine kleine Menge Xylostein, die vor längerer Zeit aus frischen Beeren dargestellt worden war, zeigte die von Hübschmann angegebenen Eigenschaften, insbesondere hervorragende Krystallisationsfähigkeit; doch lag der Schmelzpunkt wesentlich höher $\left(124^{\circ}\right)$, Hübschmann gab $\left(100^{\circ}\right)$ an.

Die Beeren gelten allgemein als giftig, werden auch, soviel wir beobachten konnten, von Vögehn nicht gefressen. Uns ist nur ein Vergiftungsfall an Menschen bekannt geworden, der sich an zwei Kindern in Mürzzuschlag (Steiermark) zutrug und unter dem Bilde einer heftigen Magendarmerkrankung verlief, obne letal zu enden. Aller Wahrscheinlichkeit nach ist das Xylostein der Träger der giftigen Eigenschaften.

Im Alkoholauszugr findet sich reichlich Anthocyan (grüne Fällung mit Bleiessig), daneben kleine Mengen eines eisengrünenden Gerbstoffes sowie organische Säuren, deren Nachweis weder bei dieser Beere noch in den folgenden Fällen durchgeführt werden konnte, da die bisherigen analytischen Methoden zur Erkennung und Trennung der Pflanzensäuren unzulänglich sind. Die neue Phenacylbromidmethode von Rather und Reid ${ }^{2}$ wurde uns erst nach Abschluß der Arbeit bekannt. Die Menge der freien Säuren, beziehungsweise der sauren Salze ist gering. Außer diesen durch Bleiessig fällbaren Stoffen sind im Alkoholauszug reichliche Mengen Zucker vorhanden, der in bekannter Weise glatt das Glukasazon lieferte (Schmelzpunkt nach zweimaligem Umkrystallisieren $205^{\circ}$ ). Die Lösung ist linksdrehend und gärfähig. Der Nachweis, daß hier Invertzucker vorliegt, wurde auf quantitativem Wege erbracht. 
$1 \mathrm{~cm}$ einer Lösung, die im $2 \mathrm{dm}$-Rohr eine Drehung von $-14 \cdot 2^{\circ}$ Ventzke zeigte, reduzierte aus Fehling'scher Lösung nach Allihn $0.1935 \mathrm{~g}$ Cu. Ist $x$ die Menge des Traubenzuckers, $y$ dic des Fruchtzuckers, so gelten annähernd die Gleichungen:

$$
\begin{aligned}
& 1.8564 x+1.7185 y=19 \cdot 35 ; x=5.250 \\
& \frac{x}{0.3268}-\frac{y}{0.1838}=-14 \cdot 2 ; y=5 \cdot 570
\end{aligned}
$$

Die beiden Zucker sind also in nahezu gleichen Mengen vorhanden.

Der wässerige Auszug der Beeren wurde zur Sirupdicke eingeengt und mit dem zwei- bis dreifachen Quantum Alkohol gefällt, wobei ein gallertartiger Niederschlag entsteht, der zur Reinigung neuerdings in heißem Wasser gelöst und unter Zusatz von etwas $\mathrm{HCl}$ wieder mit Alkohol gefällt wurde. Die gailertartige Fällung trocknete auf dem Wasserbade zu einer spröden, glasigen Masse ein. Diese ist in heißem Wasser leicht löslich, fällbar durch Blei- und Kupferazetat, $\mathrm{NaOH}$ fällt gallertig; nach dem Ansäuern mit verdünnter $\mathrm{HCl}$ löst sich der Niederschlag nicht mehr auf, während die ursprüngliche Lösung des Kohlehydrats durch $\mathrm{HCl}$ nicht gefällt wurde. Fehling'sche Lösung wird gefällt, in der Kochhitze nur wenig reduziert (vielleicht Verunreinigungen), Jod gibt keine Farbenreaktion. Die Substanz hält hartnäckig Basen, besonders Kalk fest. Es liegt zweifeilos ein Pektin vor. Der oxydative Abbau mit $\mathrm{HNO}_{3}$ lieferte neben wenig Oxalsäure reichliche Mengen von Schleimsäure. Diese enthält noch schwer zu beseitigende Basen und muß öfters aus viel salzsäurehaltigem, siedendem Wasser umkrystallisiert werden. Sie wurde schließlich in gut ausgebildeten Krystallen vom Schmelzpunkt $204^{\circ}$ erhalten. Die Hydrolyse des Pektins mit $2 \% \mathrm{HCl}$ ergab, in bekannter Weise durchgerührt, keine krystallisierenden Hydrazone oder Osazone, so daß Mannose, Dextrose und Lävulose unter den Hydrolysenprodulten in faßbare1 Menge nicht vorhanden sein können. Hingegen liefert die Destillation des Kohlehydrats mit $\mathrm{HCl}$ (von 1.06 spezifischem Gewicht) nach Tollens deutliche Mengen von Furfurol (Prüfung mit Phloroglucin und Anilinazetat). Das native Pektin ist also wohl im wesentlichen ein Galaktopentosan, in dem die Galaktosekomponente zu überwiegen scheint. Übrigens hat auch schon Bridel (l. c.) dieses Pektin untersucht und darin Galaktose und Arabinose nachgewiesen, was durch unsere Untersuchungen bestätigt erscheint. Außerdem enthält der wässerige Auszug viel Mineralstoffe, insbesondere $K$, $\mathrm{Ca}, \mathrm{PO}_{4}$ und relativ viel $\mathrm{SO}_{4}$.

Von unlöslichen Stoffen wurde noch oxalsaurer Kalk nachgewiesen, der in Krystalldrusen im Beerenfleisch vorkommt und auf mikrochemischem Wege (Gipsbildung) identifiziert wurde. Schimper ${ }^{1}$ fand ferner in der Epidermis unter dem subepidermalen

I Schimper; Jahrb. f. Wissensch. Botanik, 16:120, 1885. 
Gewebe farblose Eiweißkrystalle in Gestalt von Spindeln und Stäbchen, die bei der Reife verschwinden und daher von uns nicht bemerkt werden konnten.

In quantitativer. Beziehung wurde folgendes festgestellt:

Der Wassergehalt der frischen Beeren beträgt $88.5 \%$ (100 $g$ frische Beeren yeben $11.4986 \%$ Trockensubstanz); das Gewichtsverhältnis der trockenen Beeren zu den trockenen Samen ist 100:26; das Gewicht einer Beere (Doppelbeere) $0 \cdot 10 \xi$, das eines Samens $0.0027 \%$. Die Analyse der Frïchte von $18.8967 \mathrm{~g}$ Trockensubstanz crgab: $1.0599 g=5.60 \%$ Petroläther- und $0.2462, g=1.31 \%$ Ätherextrakt. Nach der Äther-Petrolätherextraktion wurde die Substanz mit Wasser extrahiert und der Extrakt auf $1 \mathrm{l}$ gebracht. Extrakt in $100 \mathrm{~cm}^{3} 1.055 \mathrm{~g}=55.83 \%$. darin Extralitasche $0.0615 g=3 \cdot 250 / 0 ;$ in $300 \mathrm{~cm}^{3}$ wurden nach dem Einenger. auf etwa $50 \mathrm{~cm}^{3}$ Ansäuern mit $\mathrm{HCl}$, Fällen mit Allohol, Wägen des Niederschlages auf gewogenem Filter und Abziehen der Asche die Pektine mit $0 \cdot 1365 \mathrm{~g}=2 \cdot 410 \%$ bestimmt; $100 \mathrm{~cm}^{3}$ Lösung wurden mit $10 \mathrm{~cm}^{3}$ Bleiessig versetzt, filtriert, davon $5 \mathrm{~cm}^{3}$ nach Ausfällen des Bleis mit $\mathrm{Na}_{2} \mathrm{CO}_{3}$ zur Reduktion verwendet; es ergaben sich 0.0447 o $\mathrm{Cu} \mathrm{O}=0.0198 \mathrm{~g}=23.05 \%$ Invertzucker; $100 \mathrm{~cm}$. Extralst verbrauchten $6.9 \mathrm{~cm}^{3}$ Lauge $\left(0.00469 \mathrm{~g} \mathrm{KOH}\right.$ in $\left.1 \mathrm{~cm}^{3}\right)=1.710 / 0$ freie organische Säuren; Gerbstoffe waren nicht bestimmbar.

18.8825 o Trockensubstanz wurden ebenfalls mit Wasser extrahiert und auf $1 \mathrm{l}$ aufgefült. Davon wurden $250 \mathrm{~cm}^{3}$ nach Einengen und Fällen des Pelstins auf $100 \mathrm{~cm}^{3}$ gebracht, $7 \mathrm{~cm}^{3}$ davon $z u r$ Redultion verwendet, ergaben $0.1894 \mathrm{~g} \mathrm{Ca} \mathrm{O}=$ $0.1512 g \mathrm{Cu}=0.0795 g=24.04 \%$ Invertzucker. Dieselbe Lösung mit $\mathrm{HCl}$ (6) Stunden invertiert, ergab in $6 \mathrm{~cm}^{3} 0.1691 g^{\circ} \mathrm{Cu}=0.0708 \mathrm{~g}=24.98 \%$ Zucker; somit sind invertierbare Zucker in merklicher Menge nicht vorhanden.

$200 \mathrm{~cm}^{3}$ des ursprünglichen Extrakts verbrauchten $2.5 \mathrm{~cm}^{3}$ Lauge $\left(0.029057 \mathrm{~s}^{\circ}\right.$ $\mathrm{KOH}$ in $\left.1 \mathrm{~cm}^{3}\right)=1.930 .0$ freje olganische Säuren; $100 \mathrm{~cm}^{3}$ gaben $1.0302 \mathrm{~g}=$ $54.560 \%$ lixtrakt und $0 \cdot 062 \pm g=3 \cdot 30 \%$ Extraktasche.

Die Stickstoff bestimmung nach Kjeldahl ergab $1 \cdot 310_{0} N$, die $8 \cdot 19 \%$ Rohprotein entsprechen. (Es verbranchten $2.5204 \mathrm{~g}$ Trockensubstanz $12 \mathrm{~cm}^{3}$ Säure rom Stickstofititer 0.00275 .) 3.3305 \& Trockensubstanz lieferten 0.1621 s Asche, entsprechend $\pm .87 \%$.

Die Samen sind fettreich und enthalten keine Stärke. Es wurde hier wie in den folgenden Fällen nur das Fett näher untersucht. Das Samenöl ist rotgelb, dickfiüssig und zeigt folgende Kennzahlen: Säurezahl $16 \cdot 5$; Verseifungszahl $184 \cdot 85$; Jodzah1 132.8; unverseifbare Stoffe $4.92 \%$. Das Unverseifbare besteht aus einem Caroten (Blaufärbung mit konzentrierter $\mathrm{H}_{2} \mathrm{SO}_{4}$ ) und wachsartigen Stoffen. Phytosterine sind nur in Spuren nachweisbar.

Die Fettsäuren sind flüssig und scheiden nach längerem Stehen nur geringe Mengen fester Fettsäuren aus. Seiner hohen Jodzahl entsprechend erweist sich das Öl als ein (langsam) trocknendes. In dünner Schichte auf die Glasplatte gebracht, nimmt es bei gewöhnlicher Temperatur im zerstreuten Tageslicht innerhalb von 14 Tagen $12 \%$ Sauerstoff auf, wobei es farblos wird und schließlich einen vollkommen trockenen Film bildet.

\section{Lonicera nigra $\mathrm{L}$.}

Schwarzblaue Doppelbeeren von fad süßlichem Geschmack. Das Material war bei Mürzzuschlag (Steiermark) gesammelt worden. Über diese Beeren liegen keinerlei chemische Angaben vor. 
Die qualitative Untersuchung wurde wie bei der vorigen Art durchgeführt. Die im Petroläther löslichen Stoffe rühren größtenteils aus den Samen her, auch im Ätherauszug sind hauptsächlich Substanzen der Samen enthalten, worauf wir unten zurückkommen. Doch sei bemerkt, daß sich hier auch harzartige Stoffe vorfinden, die wahrscheiniich aus dem Fruchtfleisch oder der Fruchtschale stammen und sich dadurch trennen lassen, daß sie in kaltem Äther schwer löslich sind. Diese Harze sind rotbraune, fadenziehende Substanzen, sind in Aikohol löslich und färben sich in dieser Lösung mit $\mathrm{Fe} \mathrm{Cl}_{3}$ olivgrün, auf Ätherzusatz fallen sie aus. Die Farbenreaktion nach Storch-Morawski war negativ.

Der Alkoholauszug wurde vom Lösungsmittel befreit und mit Wasser aufgenommen, wobei sich ein rotbraunes Pulver abschied, das nach der Filtration neuerlich in Alkohol gelöst und unter Salzsäurezusatz mit Wasser gefällt wurde. Der Körper hat die Eigenschaften eines Phlobaphens, gibt in wässerig-alkoholischer Lösung mit $\mathrm{Fe} \mathrm{Cl}_{3}$ eine olivgrüne Lösung, später auch einen Niederschlag, mit Kupferazetat eine graugrüne, mit Kaliumbichromat eine braune und mit Bleiazetat eine rotbraune Fäliung. Das Phlobaphen dürfte sich wohl erst während des Trocknens oder Auskochens aus den Gerbstoffen bilden. In dem Filtrat vom Phlobaphen findet sich ein Anthokyan und ein eisengrünender Gerbstoff. Freie Säuren sind wenig vorhanden, dagegen reichliche Mengen Zucker, wahrscheinlich Invertzucker.

Im Wasserauszug wurden wie im vorigen Falle Pektine nachgewiesen, daneben Kalium- und Calciumsalze.

Bei der quantitativen Untersuchung wurde folgendes festgestellt:

Das Beerengewicht verhält sich zum Samengewicht wie $100: 17 \cdot 7$; das mittlere Gewicht einer Beere (Doppelbeere) $0.104 \mathrm{~g}$, das eines Samens $0.0025 \mathrm{~g}$. Zur Bestimmung dieser Werte wurde eine größere Menge vollkommen trockerier und gezithlter Beeren gewogen, die Beeren mit kaltem Wasser mazeriert. die Samen sorgfältig ausgelesen, getrocknet ind wieder gewogen. Die angegebenen Werte sind daher nur angenäherte.

Die wie im vorigen Falle durchgefühte Analyse von $17 \cdot 244 \mathrm{~g}$ Trockensubstanz ergab: im Petrolätherauszug $1.6847 \mathrm{~g}=9.77 \%$ im Atherauszug $0.2778 \mathrm{~g}=1.61 \%$. Der: Wasserauszug aus der angegebenen Trockensubstanz wurce auf $1 \mathrm{l}$ aufgefüllt; Gesamtextrakt von $50 \mathrm{~cm}^{3} 0.5281 \mathrm{~g}=61.25 \%$; darin Extraktasche $0.022 \mathrm{~g}=2.55 \%$; Peltine in $250 \mathrm{~cm}^{3} 0.1639 \mathrm{~g}=3.8 \%$; Zucker in $10 \mathrm{~cm}^{3}$ (100 $\mathrm{cm}^{3}$ Lösung wurden mit Bleiessig geklärt auf $110 \mathrm{~cm}^{3}$ aufgefüllt) $0.1145 \mathrm{guO}=0.0914 \xi \mathrm{Cu}=0.0476 \mathrm{~g}$ Invertzucleer $=30.37 \%$ freie organische Säuren $1.87 \%$ \% $\left(100 \mathrm{cnt}^{3}\right.$ Lösung verbrauchten $1.05 \mathrm{~cm}^{3}$ Lauge vom Titer $0.03077)$; Gerbstoffe wurden nach der bekannten Methode durch Entfernen derseiben mit Hautpulver in $100 \mathrm{~cm}^{3} 0.0799 \mathrm{~g}=4.63 \%$ gefunden; die Gesamtasche von $0.862 g$ Trockensubstanz war $0.0321 \mathrm{~g}=3.720 \%$; die Stickstoff bestimmung mach $\mathrm{Kjeldah1}$ von $1.7068 \mathrm{~g}$ Trockensubstanz ergab $1 \cdot 174 \% \mathrm{~N}=7 \cdot 3380 \%$ Rohprotein. (Es wurden $7 \cdot 29 \mathrm{~cm}^{3}$ Säure vom Stickstoffiter 0.00275 verbraucht.)

Die Samen enthalten viel Fett, hingegen keine Stärke. Das Samenöl ist grün (Chlorophyll?) und salbenartig. Es weist folgende 
Kennzahlen auf: Verseifungszah1 $156 \cdot 53$, Jodzah1 101.39, Brechungsexponent bei $23^{\circ} \mathrm{C} .=1.494$. Das Öl trocknet, wenn auch langsam, auf der Glasplatte vollkommen ein und bildet einen etwas trüben Film. Die Gewichtszunahme betrug innerhalb 14 Tagen $8.18 \%$. Der Gehalt an unverseifbaren Stoffen ist sehr hoch, $21.59 \%$. Es handelt sich um wachsartige und namentlich um phytosterinartige Körper; letztere wurden durch die bekannte Reaktion nach Liebermann und Hesse-Salkowski nachgewiesen. Die Fettsäuren sind grün und salbenartig, fest, im Gegensatz zu den anderen Samenfetten, die flüssig sind.

Der Ätherauszug der Samen ist grün und reich an Phytosterinen, die durch Umkrystallisieren aus wässerigem Alkohol mit Tierkohle gereinigt werden können.

\section{Viburnum opulus L.}

Rote Beeren von saurem Geschmack und auffallendem Fettsäuregeruch. Die Beeren wurden in Mürzzuschlag (Steiermark) gesammelt. Sie sind nicht giftig.

Schon Chevreul ${ }^{1}$ und $\mathrm{Leo}^{2}$ haben darin Isovaleriansäure (Viburnumsäure), Essigsäure (?) und einen roten Farbstoff gefunden. Nach unseren Beobachtungen liegt tatsächlich ein Gemisch von freien flüchtigen Fettsäuren vor, dessen Hauptbestandteil Isovaleriansäure sein dürfte. Daneben sind wohl auch flüchtigere Fettsäuren (Essigsäure) vorhanden.

Der Petrolätherauszug der Beeren, der zum größten Teil aus den Samen stammt, wird bei diesen besprochen werden.

Der Ätherauszug, der sehr reichlich ist, rührt meistenteils vom Wachsüberzug der Fruchtschale her. Er bildet nach öfterem Umkrystallisieren aus Alkohol eine weiße, krystallinische Masse, die unter dem Mikroskop undeutliche blattartige Gebilde darstellt: die Phytosterinreaktion war negativ. In den Mutterlaugen verbleibt ein rotgelbes Harz, das auch in Äther-Alkohol leicht löslich ist, zum Unterschied von den Harzen der übrigen Beeren. Es bildet nach Beseitigung des Lösungsmittels eine klebrige, fadenziehende Masse, gibt aber nicht die Storch-Morawski'sche Harzreaktion.

Im Alkoholauszug wurde wie bei der vorausgehenden Art ein rotbraunes Phlobaphen gefunden, im Filtrat Gerbstoffe. Diese gaben mit Eisenchlorid eine dunkelgrüne Färbung und einen schwarzgrünen Niederschlag, mit Kupferacetat einen grünen, mit Kaliumbichromat einen rotbraunen und mit Bleiazetat einen gelben; mit Kochsalzgelatinelösung eine bräunliche, klumpige Ausscheidung. Der Alkoholextrakt enthält ferner bedeutende Mengen organischer Säuren, von deren Trennung aus den früher angeführten Gründen

1 Chevreu1, Ann. Chim., 23/22.

2 Leo, Dingl. Polyt. Joum., 1832, 46,120. 
abgesehen wurde. Der Extrakt ist reich an Zucker; er liefert ein Osazon, das nach nehrmaligem Umkrystallisieren den Schmelzpunkt $204^{\circ}$ zeigte. Der quantitative Nachweis ergab wieder das Vorhandensein von Invertzucker mit überwiegender Glukose.

Die Lösung, die im $2 d m$-Rohr $-0.8^{\circ}$ Ventzke drehte, gab bei der Redulktion mit Fehling'scher J.ösung in $1 \mathrm{~cm}^{3} 0.1855 \mathrm{~g} \mathrm{Cu}$, woraus sich aus der früher angegebenen Formel fiir $x=6 \cdot 48$, für $y=3 \cdot 79$ in $100 \mathrm{~cm}^{3}$ ergibt.

Aus dem konzentrierten Extrakt krystallisierte die Glukose nach längerem Stehen aus und konnte nach mehrmaligem Umkrystallisieren aus Alkohol rein erhalten werden.

Der Wasserauszug ergab Pektine, die wie bei den vorigen Arten die Furfurolreaktion nach Tollens und nach dem oxydativen Abbau mit $\mathrm{HNO}_{3}$ Schleimsäure vom Schmelzpunkt $204^{\circ}$ lieferten. Kalium- und Calciumsalze sind ebenfalls im Wasserauszug vorhanden.

Quantitativ ergibt sich: Wassergehalt der frischen Beeren $84.92 \%$; Beerengewicht zum Samengewicht $100: 27 \cdot 5$; in $16.4700 \mathrm{~g}$ Trockensubstanz Petrolätherauszug $1.3468 \mathrm{~g}=8.17 \%$; Ätherauszug $1.0885 \mathrm{~g}=6.60 \%$; dieselbe Trockensubstanz mit Wasser auf $1 \mathrm{l}$ gebracht, davon in $100 \mathrm{~cm}^{3}$ Gesamtextrakt $0.8094 \mathrm{~g}=$ $49 \cdot 14 \%$; darin Extraktasche $0.0376 \mathrm{~g}=2.28 \%$; Pektine in $250 \mathrm{~cm}^{3} 0.221 \mathrm{~g}=$ $5.36 \%$; freie organische Säuren $5 \%\left(2.8 \mathrm{~cm}^{3} \mathrm{n}_{2} \mathrm{Na} \mathrm{OH}\right.$ vom Faltor 1.0506 für $100 \mathrm{~cm}^{3}$ Lösung), Gerbstoffe in $100 \mathrm{~cm}^{3} 0.0489 \mathrm{~g}=2.97 \%$. In $8.822 \mathrm{~g}$ Trockensubstanz: Petrolätheratiszug $0.8487 \mathrm{~g}=9.620_{0}^{\prime}$; Ätherauszug $0.4435 \mathrm{~g}=5.020 \%$; der Wasserauszug derselben Trockenprobe auf $1 / 2 \mathrm{l}$ gebracht, in $50 \mathrm{~cm}^{3}$ Gesamtextrakt $0.4575 \mathrm{~g}=51.85 \%$; Extraktasche darin $0.0200 \mathrm{~g}=2.267 \%$; Pektine in $200 \mathrm{~cm}^{3} 0.1852 \mathrm{~g}=5.25 \%$ Zucker in $10 \mathrm{~cm}^{3}$ (100 $\mathrm{cm}^{3}$ Lösung mit Bleiessig geklärt, auf $110 \mathrm{~cm}^{3}$ gebracht) $0.123 \mathrm{~g} \mathrm{CuO}=0.0982 \mathrm{~g} \mathrm{Cu}=0.0512 \mathrm{~g}$ Invertzucker $=31.92 \%$; Gerbstoffe in $50 \mathrm{~cm}^{3} 0.028 \mathrm{~g}=3.17 \%$; die Gesamtasche von $0.8822 g$ Trockensubstanz $=0.0305 \mathrm{~g}=3.46 \%$. Der Stickstoffgehalt nach Kjeldahl $1.115 \%=7 \cdot 22 \%$ Rohprotein, (Bei $1.7644 g$ Trockensubstanz wurden $7 \cdot 41 \mathrm{cnt}^{3}$ Säure rom Stickstofftiter 0.00275 verbraucht.)

Die Samen sind fettreich $(21 \%) \cdot$ und enthalten keine Stärke. Das reine Samenöl ist goldgelb, während das aus den ganzen Früchten gewonnene rotgelb ist. Das Öl gibt keine Carotenreaktion und zeigt folgende Kennzahlen: Verseifungszah1 192.3, Jodzahl 108, Brechungsexponent 1.484 bei $23^{\circ}$; spezifisches Gewicht bei $15^{\circ} \mathrm{C} .=0.9252$; unverseifbare Bestandteile $2.34 \%$. Das Öl trocknet auch nach längerer Zeit nicht vollständig auf der Glasplatte ein. Das Unverseifbare besteht aus einem Lipochrom neben wachsartigen Körpern; die Phytosterinreaktionen waren negativ.

\section{Viburnum Lantana $L$.}

Die Früchte sind unreif grün, halbreif rot, ausgereift schwarzblau, haben süßlichen Geschmack und sind nicht giftig.

Sie wurden in Mürzzuschlag (Steiermark) gesammelt. Bisher sind sie chemisch nicht untersucht worden. 
Der reichliche Petrolätherauszug stammt größtenteils aus den Samen und wird bei diesen besprochen.

Der Ätherauszug besteht aus Phytosterinen und wachsartigen Körpern, daneben enthält er auch ein Harz, das die gleichen Eigenschaften zeigt wie die anderen in dieser Gruppe gefundenen und das durch Äther-Alkohol fällbar ist.

Aus dem Alkoholauszug ließ sich ein hellbraun gefärbtes Phlobaphen isolieren, das aber beim Trocknen stark nachdunkelte. Gerbstoffe sind nur in geringer Menge vorhanden, ebenso freie organische Säuren. Der Nachweis des Invertzuckers geschah wie in den früheren Fällen durch Darstellung des Osazons und durch die Bestimmung des Reduktionsvermögens und der Polarisation.

Xach der schon frihher angefïhrten Reclinung ergab sich:

J. Bestimmung: Polarisation im 2 am-Rohr $+11^{\circ}$ Ventzke, Reduktion $0 \cdot 151$ \& Cu in $1 \mathrm{~cm}^{3}, x=6.580, y=1.680 ; 0$.

II. Bestimmung: Polarisation in 2 am-Rohr $+8^{\circ}$ Ventzke, Redultion 0.11952 is Cu in $1 \mathrm{~cm}^{3}, x=5 \cdot 14 \%, y=1.400 \%$.

Die Glukose überwiegt auch hier, krystallisierte nach längerem Stehen aus und wurde durch öfteres Umlösen aus siedenden Holzreist rein erhalten.

Im Wasserauszug konnte ein Pektin in der gleichen Weise wie früher (oxydativer Abbau zu Scbleimsäure, Pentosennachweis nach Tollens) nachgewiesen werden. Daneben scheinen noch andere Polysaccharide vorhanden zu sein.

\section{Die quantitative Bestimmung ergab:}

Beerengewicht zum Samengewicht wie 100: 48 ; das mittlere Gewicht cineBeere $0.054 \mathrm{~g}$, das eines Samens $0.026 \mathrm{~s}$. Von $16.352 \mathrm{~s}$ Trockensubstanz $1.503 \mathrm{~s}=$ (). $19 \%$ Petrolätherauszug, $0.0769 g=0.47 \%$ Ätherauszug. Wasserauszug derselben Trockensubstanz auf $1000 \mathrm{~cm}^{3}$ gebracht, in $100 \mathrm{~cm}^{3} 0.6580 \mathrm{~s}=40 \cdot 24 \%$ Gesamtextralkt; darin Extraktasche $0.043 \mathrm{~g}=2.620 \%$; Pelitine in $250 \mathrm{~cm}^{3} 0.0851 \mathrm{~s}=$ $2.0800_{0}$; Zucker in $10 \mathrm{~cm}^{3}\left(100 \mathrm{~cm}^{3}\right.$ Lösung mit Blciessig geklärt, auf $110 \mathrm{~cm}^{35}$ (sebracht) $0.0743 \mathrm{~g} \mathrm{Cu}=0.03905 \mathrm{~g}$ Invertzucker $=26.27 \% \%$ freic organische Säuren $1.2600_{0}\left(0.7 \mathrm{~cm}^{3} \mathrm{n} / 2\right.$ KOH vom Faktor 1.0506 für $100 \mathrm{~cm}^{3}$ l.ösung); Gerbstoffe $0.0133, s=0.81 \%$; Gesamtasehe von $1.635 \%$ \& Trockensubstanz $0.0572 g=$ $3 \cdot 49 \%$; die Sticlsstoff bestimmung nach Kjeldahl ergab $1.04 \% \% \mathrm{~N}=6.50 \%$ Rollprotein $\left(6 \cdot 144 \mathrm{cmh}^{3}\right.$ Säure vom Sticlsstofftiter $0.0027 \bar{z}$ für 1.6239 \& Trockensubstanz).

$2 \cdot 6871 g$ Trockensubstanz gaben $0.2786 g=10 \cdot 3 \%$ an Petroläther und 1) $.0141 \mathrm{~g}=0.5 \%$ an Äther lösliche Stoffe ab. $16.0235 \mathrm{~g}$ Trockensubstanz mit Wasser extrahiert und auf $1000 \mathrm{~cm}^{3}$ gebracht, enthielten in $100 \mathrm{~cm}^{3} 0.6262 \mathrm{~g}=$ $39.08 \%$ Gesamtextrakt und $0.0403 g=2 \cdot 50 ! 0$ Extraktasche darin; Pektin in $300 \mathrm{~cm}^{3} 0.1877 \mathrm{~g}=3.8 \% \% 100 \mathrm{~cm}^{3}$ Lösung mit Bleiessig geklärt und auf $110 \mathrm{~cm}^{3}$ yebracht, ergaben in $25 \mathrm{~cm}^{3} 0.202 \mathrm{~g} \mathrm{CuO}=0.0848 \mathrm{~g}=23.3 \%$ Invertzucker; freie organische Säuren in $100 \mathrm{~cm}^{3} 1.8 \%$ (100 $\mathrm{cm}^{3}$ Lösung verbrauchten $0.6 \mathrm{~cm}^{3}$ ${ }^{\prime \prime}: \mathrm{KOH}$ von Faktor 0.969488$)$; Rohasche von $1.452 \mathrm{~g}$ Trockensubstanz $0.0499 \mathrm{~g}=$ $3 \cdot 4 \%$; der Stickstoffgehalt nach $\mathrm{Kjeldahl} 1.220 \% \mathrm{~N}=7.63 \%$ Rohprotein $11: 3495 \mathrm{~g}$ Trockensubstanz verbrauchten $2 \cdot 35 \mathrm{~cm}^{3} \mathrm{n} / \mathrm{g}$ Schwefeisäure); $3 \cdot 5736 \mathrm{~g}$ Trockensubstanz lieferten $1.4686 \mathrm{~g}$ Rohfaser, entsprecliend $41.09 \%$. 
Das Samenöl ist grüngelb, gibt keine Carotenreaktion und zeigt folgende Kennzahlen: Verseifungszahl $192 \cdot 5$, Jodzahl $120 \cdot 98$; Brechungsexponent bei $23^{\circ} 1.486$, die Gewichtszunahme des Öls in dünner Schichte auf der Glasplatte betrug $7 \cdot 33 \%$. Das Trockenwerden erfolgte aber erst nach sehr langer Zeit. Das Unverseifbare bestand aus Phytosterinen und wachsartigen Körpern.

\section{Sambucus nigra $L$.}

Die Beeren dieser. Art sind früher offizinell und daher schon einigemale Gegenstand chemischer Untersuchung gewesen.

Nach älteren Angaben von $\mathrm{En} Z^{1}$ enthalten sie geringe Mengen eines ätherischen Öls, Apfel- und Weinsäure, keine Zitronensäure, Spuren flüchtiger Säuren, Wachs, Zucker, Gerbstoff und Anthokyan. Nach neueren Untersuchungen von $\mathrm{Adam}^{2}$ ist jedoch weder Apfel- noch Weinsäure vorhanden. Derartige Widersprüche sind auf die Mangelhaftigkeit der analytischen Methoden zurüickzuführen. auf die bereits eingangs hingewiesen worden ist. Tollens und Sack ${ }^{3}$ geben das Vorkommen von Tyrosin an; Em. Bourquelot und Em. Danjou, ${ }^{4}$ die sich viel mit dem in der Rinde und in den Blättern von Sambucus nigra vorkommenden Glukosid beschäftigten, fanden dieses auch in den grünen Früchten und in diesen auch geringe Mengen von Invertin und Emulsin; Guignard ${ }^{5}$ bestätigt, daß die Früchte Glukosid enthalten, nur so lange sie grün sind; der frische Saft der reifen Früchte enthält kein Glukosid und kein Emulsin, während sich letzteres in den reifen Samen tindet. E. Couperot ${ }^{6}$ findet auch in den grünen Früchten und Blättern Glukosid und Nitrat, welche beide beim Trocknen verloren gehen. Einige wenige quantitative Angaben stammen von Wittmann ${ }^{7}$ her.

Wie bereits erwähnt, lag gekauftes Material vor, über dessen Alter wir nichts angeben können.

Der Petrolätherauszug rührt rom Samenfett her, das später besprochen wird.

Der Ätherauszug ist grünlich, krystallinisch und ergab nach mehrmaligem Umkrystallisieren aus wässerigem Alkohol und Tierkohle Phytosterine und wachsartige Körper, neben einem gelbbraunen Rückstand, der wegen seiner geringen Menge nicht näher untersucht werden konnte. Harz wurde in Ätherextrakt nur in kleiner Menge gefunden.

1 Enz, Vierteljalursch. f. prakt. Pharm., $1859 / 311$.

2 Zeitschrift des österr. Apoth.-Vereins, 1906/243.

3 Berliner Berichte, 1904/4115.

\pm Chem. Zentralblatt, 1905/II, 1267.

3 Chem. Zentralblatt, 1906/I, 374; Compt. rend, 19051193.

"Chem. Zentralblatt, 1909/1, 864.

7 Zeitschrift des landw. Versuchswesens in Öst., 1901/131. 
Der Alkoholauszug ergibt nichts von den früheren Fällen Verschiedenes. Er enthält nur Spuren eines Phlobaphens, etwas Harz, reichlich Anthokyan, ferner Gerbstoffe, freie organische Säuren und Zucker (wahrscheinlich Invertzucker).

Der Wasserauszug enthält Pektin, daneben im Verhältnis $z u$ den anderen Beeren dieser Gruppe bedeutende Mengen von Mineralsalzen, besonders $\mathrm{K}, \mathrm{Ca}, \mathrm{PO}_{4}$.

Die quantitative Analyse ergab:

Beerengewicht zum Samengewicht wie 100:40. Das Gewicht eines Samens 1). (1)32 $\%$, das einer Beere $0.0252 \xi .18 \cdot 160$ s Trockensubstanz ergahen: Petrolitherauszug $2 \cdot 1466 g=11 \cdot 81 \%$; Ätherauszug $0.2501 g=1 \cdot 37 \%$; der Wasserauszug derselben Trockenprobe wurde auf $1000 \mathrm{~cm}^{3}$ gebracht und ergab: In $100 \mathrm{~cm}^{3}$ $0.8172 g=450 \%$ Gesamtextrakt, $0.115 g=6.33 \%$ Extralktasche darin; Pektine in $250 \mathrm{~cm}^{3} \quad 0.1590 \mathrm{~g}=3.51 \%$; Zucker in $25 \mathrm{~cm}^{3} \quad 0.2142 \mathrm{~g}$ Cu O $=0.171 \mathrm{~s}$

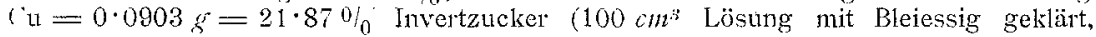
auf $110 \mathrm{~cm}^{3}$ gebracht); Gerbstoffe in $100 \mathrm{~cm}^{: 3} 0.053 \mathrm{~g}=2.910 ;$ freie organische Säuren $3.050 / 0\left(0.9 \mathrm{~cm}^{3} \mathrm{KOH}\right.$ vom Titer 0.03077 für $50 \mathrm{~cm}$ : Lösung); die Gesamtasehe für $1.816, g$ Trockensubstanz $0.134 g=7.37 \%$. Der Stickstoff nach Kjeldahl wurde mit $2.890^{\prime}=18.060 \%$ Rohprotein gefunden $(1.816 \mathrm{~g}$ Trockensubstanz verbrauchten $19.09 \mathrm{~cm}^{3}$ Säure vom Stickstofftiter 0.00275 ). Nach einer zweiten Bestimmung wurde der Stickstoff mit $2.99 \%=18.68 \%$ Rohprotein festgestelit $\left(0 \cdot 908\right.$ is Trockensubstanz verbrauchten $\left.3.88 \mathrm{~cm}^{3} \cdot \mathrm{n}_{2} \mathrm{H}_{22} \mathrm{SO}_{4}\right)$.

Der Stickstoffgehalt erscheint selr hoch und fällt aus dem Rahmen des Gesamtbildes heraus, um so mehr, da das relative Samengewicht nicht auffallend lneh ist. Über den Grund dieser: Tatsache können wir vorläufig nichts angeben. $4 \cdot 156, \xi$ Trockensubstanz lieferten $1 \cdot 2746 \xi$ Rohfaser.

Die Samen sind sehr ölreich und wurden deshalb in der Kriegszeit zur Ölgewinnung in Betracht gezogen. Die Angaben über den Fettgehalt sind widersprechend. Nach den Mitteilungen des deutschen Kriegsausschusses für Öle und Fette ${ }^{1}$ enthalten die Beeren in der Trockensubstanz bloß $6.1 \%$, nach Angaben von Muth 2 hingegen $18 \cdot 7 \%$, während die Samen $30 \%$ enthalten sollen. Wir fanden in den ganzen Früchten $13 \%$ Ätherauszug.

Das Öl ist braungrün, von auffallend geringer Viskosität und besitzt folgende Kennzahlen: Verseifungszahl $190 \cdot 05$, Jodzahl $157 \cdot 78$, Brechungsexponent bei $23^{\circ} 1.485^{\circ}$; das spezifische Gewicht bei $15^{\circ}=0.9407$; die Gewichtszunahme auf der Glasplatte betrug innerhalb von 14 Tagen $15 \cdot 11 \%$, wobei das Öl einen vollkommen trockenen, klaren Film bildete. Das Unverseifbare betrug $4.52 \%$ und enthielt neben einem gelben Körper (Lipochrom) hauptsächlich Phytosterine und wachsartige Körper.

\section{Sambucus racemosa $L$.}

Das Material war in Mürzzuschlag (Steiermark) gesammelt worden. Die Beeren sind ziegelrot, von saurem Geschmack. Zum Unterschied von allen anderen untersuchten Beeren enthält neben

1 Zeitschr. für angew. Chemie, 30/16 (1917).

- Jahresbericht der Vereinigung für angew. Botanik, $1917 / 14$ u. 36 . 
den fettreichen Samen auch das Fruchtfleisch beträchtliche Mengen von fettem Öl. Dieses ist zuerst von Zellner ${ }^{1}$ untersucht worden. In der Kriegszeit hat man diesen Fruchtölen mehr Aufmerksamkeit geschenkt, doch scheint es sich teilweise um Mischöle (Samenund Fruchtfleischöl) gehandelt $z u$ haben, wie aus den angegebenen Werten und der Gewinnungsweise zu schließen ist. ${ }^{2}$

Die Beeren sind nicht giftig und wurden während des Krieges korbweise auf Marmelade verarbeitet, ohne daß ein Vergiftungsfall bekannt geworden wäre; nach anderen Angaben soll aber das aus den Beeren gewonnene Öl schädlich sein. Es wurde deshalb auf Nitrilglukoside geprüft, doch war Blausäureabspaltung nicht nachweisbar. Die Annahme, daß es sich um giftige, N-haltige Glukoside handeln könnte, war naheliegend, da im Laub und in der Rinde von Sambucus nigra ein solcher Stoff (Sambunigrin) von Bourquelot und Danjou ${ }^{3}$ nachgewiesen worden ist, doch hatte weder dieser noch Guignard ${ }^{4}$ im Laub und in der Rinde von Sambucus racemosa einen solchen Körper nachweisen können.

Der Alkoholauszug enthält einen Anthokyanfarbstoff von eigentümlicher Nuance, der die ziegelrote Farbe der Beeren bedingt, vielleicht spielt aber auch die gelbe Farbe des Fruchtfleischöles mit, das in feinen Tropfen im Fruchtfleisch verteilt ist, so daß der Saft als gelbe Emulsion erscheint. Im übrigen gibt der Farbstoff die Anthokyanreaktionen, und zwar mit Bleiacetat eine grüne Fällung, mit $\mathrm{NH}_{3}$ eine blaue, mit Mineralsäuren eine karmosinrote Färbung. Gerbstoffe sind nur in geringer Menge vorhanden, dagegen bedeutende Mengen freier organischer Säuren. Der Nachweis des Invertzuckers geschah wie in den vorigen Fällen durch Darstellung des Osazons und mittels der quantitativen Methode, die wieder ein Überwiegen an Glukose ergab.

Polarisation im $1 d n$-Rohr $-1 \cdot 2^{\circ}$ Ventzke, Reduktion von $2 \mathrm{cn}{ }^{3}$ Lösung 1) $.2387 \mathrm{~g}$ Cu $\mathrm{O}=0.1907 \mathrm{~g}$ Cu; somit in $100 \mathrm{~cm}^{3}$ Lösung $2 \cdot 1$ ? Lärulose und $3 \cdot 1$ Dextrose.

Im Wasserauszug findet sich ein Pektin, das ähnliche Eigenschaften wie das von Lonicera und Viburmum zeigte und nicht näher untersucht wurde, ferner bedeutende Mengen von Mineralstoffen.

1 Monatshefte, 1902 u. $1917 / 87$.

W. Normann,.. Zeitschr. f. angew. Chemie, 1916/I, 338; R. Prasch, Obstkemöle, 85; F. Mach, Badische landw. Wochenschr., 1916; M. Thoms, Ber. der deutschen phar. Ges., 1920, 29/598; Matthes u. Rossié, Arch. d. Pharm., 1918, 256/284; Chem. Umschau, $1920 / 126$.

3 Bourquelot und Daujou, Compt. rend., 1908/598; Chem. Zentralbl, $1905 /$ III, 1267.

^Guignard, Compt. rend., $1905 / 16$. 


\section{Die quantitative Analyse ergab:}

Wassergehalt der frischen Beeren $83 \cdot 240 \%$. Gewichtsverhältnis der Samerr $z u$ den Beeren wie 100:52. Beerengewicht $0.01052 s$, Samengewicht $0.0018 g$; $24 \cdot 2213 g$ Trockensubstanz nach der Entfettung mit Wasser extrahiert und auf $1 l$ gebracht, ergaben: Peltine in $300 \mathrm{~cm}^{5} 0.2019 \mathrm{~g}=2.78 \%$; Extralit in $100 \mathrm{~cm}^{\text {st }}$ $0.6688 g=27 \cdot 61 \%$; Extraktasche darin $0.094 \mathrm{~g}=3.88 \%$; $100 \mathrm{~cm}^{3}$ Lösung mit I3leiessig geklärt und auf $110 \mathrm{~cm}^{3}$ gebracht, in $20 \mathrm{cmo}^{3} 0.0627 \mathrm{~g}^{\mathrm{Cu}}=7.790 \%$ Invertzucker; freie organische Säuren $3.17 \%\left(2.5 \mathrm{en} \mathrm{n}^{3} \mathrm{KOH}\right.$ vom Titer 0.03077 für $100 \mathrm{~cm}^{3}$ Lösung). Der Stickstoff nach $\mathrm{Kjeldahl}$ betrug $1.607 \%=10.04 \%$ Rohprotein $\left(0.9232 \mathrm{~g}\right.$ Trockensubstanz verbrauchten $2.12 \mathrm{~cm}^{3} \mathrm{n} / 2$ Schwefelsäure); der Stickstoff nach Stutzer; $1.8464 \mathrm{~g}$ Trockenstibstanz verbrauchten $3.78 \mathrm{~cm}^{\text {ir }}$ $n_{2}$ Schwefetsäure, daher $\mathrm{N}=1.43 \%$, entsprechend $8.95 \%$ Reinprotein. $3.277 \delta$ Trockensubstanz gaben $1.0224 g=31.20 \%$ Rohfaser; $3.6928 g$ Trockenstbstanz. $0.1917 g=5.19 \%$ und $1.6967 \mathrm{~g}$ Trockensubstanz $0.0855 g=5.04 \%$ Asche.

$22 \cdot 2426 \mathrm{~g}$ mit Wasser extrahiert und auf $500 \mathrm{~cm}^{3}$ gebracht, ergaben: Extrakt: in $100 \mathrm{~cm}^{3} 1.3096 \mathrm{~g}=29 \cdot 43 \% ; 100 \mathrm{~cm}^{3}$ Lösung auf $125 \mathrm{~cm}^{3}$ angefüllt und $50 \mathrm{~cm}^{3}$ davon mit Fehling'scher Lösung zur Reduktion gebracht, ergaben 0.291 s $\mathrm{Cu}=0.158 \mathrm{~g}$ Invertzucker $=8.88 \% ; 100 \mathrm{~cm}^{3}$ enthielten $4.6 \%$ freje organische Säuren ( $7 \mathrm{~cm}^{3} \mathrm{~m} / 2$ Lauge vom Faktor $1 \cdot 0442$ wurden verbraucht).

Die Samen enthalten $27 \%$ fettes Öl in der Trockensubstanz ungefähr ebensoviel wie das trockene Fruchtfleisch. Das Samenöl ist bereits von Zellner (1. c.) untersucht worden.

\section{Symphoricarpus racemosa L.}

Diese Beeren sind bereits mehrfach untersucht worden, und zwar von Hermann und Tollens, ${ }^{1}$ die darin Dextrose 5 bis $9 \%$ und ebensoviel Lävulose, Gummi und Eiweiß fanden, ferner von Bride1 ${ }^{2}$ und von C. B. Smith, ${ }^{3}$ der die Asche quantitativ untersucht hat und im übrigen $17 \cdot 17 \%$ Zucker fand.

Unser Untersuchungsmaterial stammte aus dem botanischen Garten der Wiener Universität.

Die Beeren sind weiß und besitzen einen fad süßlichen Geschmack.

Im Petrolätherauszug findet sich ein fettes Öl, meistens aus den Samen, das bei diesen zur Besprechung gelangt.

Im Ätherauszug waren kleine Mengen von Phytosterinen und wachsartigen Körpern vorhanden.

Der A1koholauszug enthält sehr wenig eisengrünende Gerbstoffe, Anthokyan fehlt vollständig; dagegen ist der Zuckergehalt sehr hoch. Die Beeren schmecken getrocknet fast wie Feigen, haben aber einen unangenehmen Beigeschmack. Unser Befund steht in Übereinstimmung mit den Angaben von Tollens und im Gegensatz.

1 Annalen der Chemie, $1885 / 50$.

: Journ. de Pharm. et Chimie, $1907 / 536$.

3 Chem. News, 107/226 (1913). Diese Arbeit macht keinen ganz vertrauenswärdigen Eindruck. 
zu B. Smith, der einen relativ niedrigen Zuckerwert angibt. Der Zucker ist Invertzucker und ergab:

Polarisation im $2 d m$-Rohr $-2 \cdot 5^{\circ}$ Ventzke Redulition 0.1654 g Cu; $2 \cdot 380$ Dextrose und $2 \cdot 350 / 0$ Lävulose.

Im Wasserauszug wurde wieder Pektin und Mineralstoffe wie in den übrigen Beeren gefunden.

Die quantitative Analyse ergab:

Verhältnis des Beeren- zum Samengewicht wie 100:13; das mittlere Gewicht einer Beere 0.1007 of das eines Samens $0.0061 g$; der Wassergehalt war $z u$ $80 \cdot 360_{0}^{0}$ bestimmt worden.

$6.5396 \mathrm{~g}$ Trockensubstanz gaben 0.2435 \& Petrolätherauszug $=3.720 \%$ und $0 \cdot 1575 g=2 \cdot 41 \%$ Ätherauszug. $21 \cdot 7862 g$ Trockensubstanz mit Wasser extrahiert und auf $1000 \mathrm{~cm}^{3}$ gebracht, ergaben: In $100 \mathrm{~cm}^{3}$ Extrakt $1.4588 \mathrm{~g} \doteq 74.58 \%$ Extraktasche $0.0600 \mathrm{~g}=2 \cdot 75 \%$; Pektin in $300 \mathrm{~cm}^{3} 0.1682 \mathrm{~g}=2 \cdot 570 \% 100 \mathrm{~cm}^{\text {* }}$ lösung mit Bleiessig geklärt und auf $110 \mathrm{~cm}^{3}$ gebracht, in $20 \mathrm{~cm}^{\circ} 0.3793 \mathrm{~g} \mathrm{Cu}=$ $0.2118 \mathrm{~g}$ Invertzucker $=53.47 \% ; 200 \mathrm{~cm}^{\circ}$ Lösung geklärt und auf $50 \mathrm{~cm}^{*}$ eingeengt, davon $2 \mathrm{~cm}^{3}$ zur Redulation verwendet, lieferten $0.180 \mathrm{~g} \mathrm{Cu}=0.0973 \mathrm{~s}$ Invertzucker, entsprechend $56 \%$; Gerbstoffe für eine quantitative Bestimmung zu wenig. Freie organische Süuren $1.270 \%\left(100 \mathrm{~cm}^{3}\right.$ verbrauchten $0.9 \mathrm{~cm}^{3} \mathrm{KOH}$ vom Titer $0.003077 \mathrm{~g}$ ).

$3.4877 \mathrm{~g}$ Trockensubstanz verbrauchten bei der Stickstoff bestimmung nach Kjeldahl $8.28 \mathrm{~cm}^{3} \mathrm{H}_{2} \mathrm{SO}_{1}$ vom Stickstofftiter $0.00275 ; \mathrm{N}=0.650 !=4.060 !$ Rohprotein.

$2.9231 g$ gaben $0.0914 g$ Asclie $=3.12 \%$ auf Trockensubstanz bezogen.

Das Samenöl ist blaßgelb, dickflüsșig und zeigt die Jodzahl 131.8. Die Trockenprobe ergab innerhalb von sechs Wochen die Gewichtszunahme $5 \cdot 83 \%$, das Öl war nach dieser Zeit noch nicht ganz trocken.

Der Übersicht halber sind die erhaltenen Resultate in der Tabelle I zusammengefaßt.

Aus dem gesamten Zahlenmaterial scheint sich eine Tatsache $z u$ ergeben, die auch sonst auf dem Gebiete der Pflanzenchemie $\mathrm{zu}$ beobachten ist (soweit das bisherige Material zu beurteilen gestattet) und sich etwa folgendermaßen aussprechen läßt: Es liegt ein allgemeiner Typus der Zusammensetzung vor, wie er sich in ähnlicher Art auch bei den Beerenfrüchten aus anderen Familien, $z$. B. der Rosaceen und Ericaceen, vorfindet.

Dieser Grundtypus läßt sich in unserem Falle etwa folgendermaßen charakterisieren: Wassergehalt der frischen Beeren $83 \%$, in der Trockensubstanz durchschnittlich $50 \%$ Wasserextrakt, $25 \%$ Invertzucker mit häufig überwiegender Glukose, $3 \%$ Pektine, $3 \%$ freie Säuren (als $\mathrm{KOH}$ berechnet), $3 \%$ wasserlösliche Mineralstoffe, $4 \%$ Gesamtasche, $7 \%$ Proteine, Gerbstoffe in geringer Menge, oft nicht quantitativ bestimmbar, $1.5 \%$ wachsartige Stoffe der Beerenschale. Verhältnis von Beeren- zu Samengewicht wie 100:32. Allerdings finden sich bei einigen der obigen Analysen sehr große Abweichungen von den Mittelwerten; allein es besteht kein 


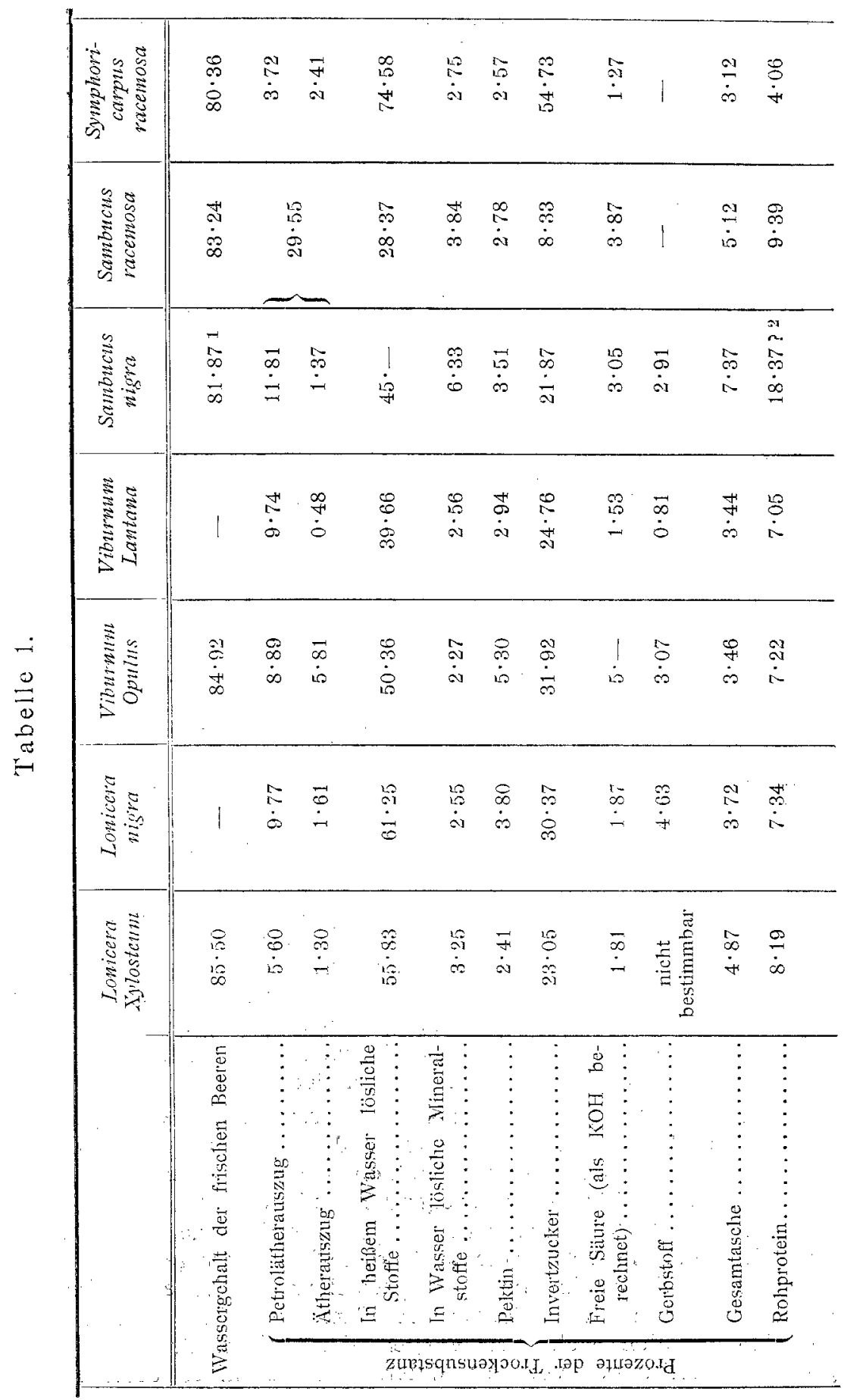




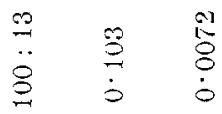

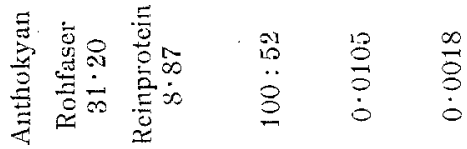

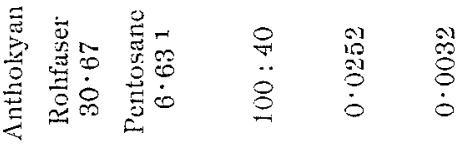

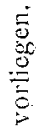

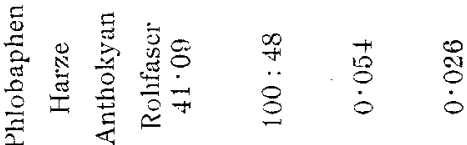

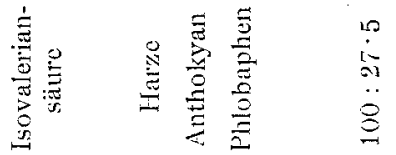

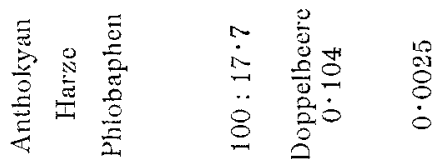

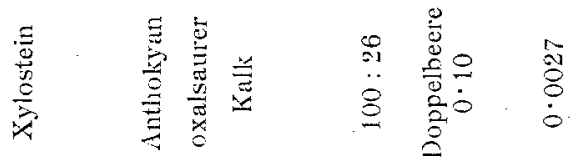

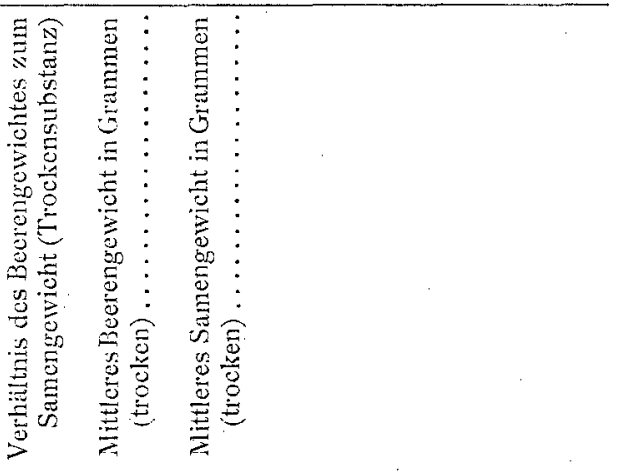


Zweifel, daß, wenn noch mehr Analysen verschiedener Caprifoliaceenbeeren vorlägen, diese Abweichungen um so seltener auftreten würden, je größer sie sind (wie die Ausreißer beim Trefferbild einer Schießscheibe). Dazu kommt noch, wie schon eingangs bemerkt, der Umstand, daß die Untersuchung der ganzen Früchte eine Verschiebung der Zahlenwerte hinsichtlich der löslichen Stoffe zur Folge hat, so daß die obigen Zahlen, soweit es sich um die Zusammensetzung des Fruchtfleisches handelt, eigentlich nicht unmittelbar vergleichbar sind. Diese Verschiebung hängt von dem Verhältnis des Beeren- zum Samengewichte $a b$ und deshalb wurde dieses Gewichtsverhältnis in allen Fällen bestimmt, um die $\mathrm{Zu}$ sammensetzung des samenfreien Beerenfleisches wenigstens ungefähr berechnen zu können. Dabei müssen freilich die beiden nicht genau zutreffenden Annahmen gemacht werden, daß die Samen bei der Wasserextraktion keinen merkbaren Substanzverlust erleiden und daß der Petrolätherauszug ausschließlich die Samenöle enthält. Die Fehler der ersten Annahme machen höchstens einige wenige Prozente aus, hingegen muß in dem Falle von Sambucus racemosa, wo auch das Fruchtfleisch viel Fett enthält, dieses letztere (mit etwa der Hälfte des Gesamtwertes) in die Berechnung miteinbezogen werden. Führt man diese Rechnungen aus, so bemerkt man, daß die extremen Werte der obigen Tabelle viel näher aneinanderrücken und sich den Zahlen des Grundtypus daher besser anschließen.

Aber natürlich bleiben nach dieser Umrechnung noch große Differenzen bestehen, die darin ihren Grund haben, daß in einzelnen Fällen eine ungewöhnliche Anreicherung des einen oder anderen Stoffes stattfindet, wobei die Prozentsätze der anderen Stoffe zwar gedrückt, aber in ihrem gegenseitigen Verhältnis meist wenig beeinflußt erscheinen; solche Fälle sind $z$. B. die Fettanreicherung bei Sambucus racemosa oder die Zuckerspeicherung bei Symphoricarpus racemosa. Die Verschiedenheiten, die durch das Auftreten singulärer Stoffe wie der Isovaleriansäure bei Viburmum Opulis und des Xylosteins bei Lonicera Xylosteum bedingt werden, kommen in quantitativer Beziehung kaum in Betracht. Sie haben auch in qualitativer Beziehung nur Wert für die chemische Charakterisierung der Art, nicht der Familie.

Der Stickstoffgehalt der untersuchten Beeren ist in allen Fällen mit einer einzigen Ausnahme gering; das Beerenfleisch selbst ist äußerst stickstoffarm, sein N-Gehalt wird einige Zehntelprozente kaum übersteigen; der $\mathrm{N}$-Gehalt der ganzen Beeren rührt daher überwiegend von den Samenproteiden her und zeigt im allgemeinen Werte von 1 bis $1.5 \%$ mit Ausnahme von Sambucus nigra.

In der Tabelle 2 ist eine Übersicht über die untersuchten Samenöle enthaiten.

Sie weisen eine recht bemerkenswerte Übereinstimmung auf, die Übereinstimmung der Zahlen tritt erst bei der Umrechnung auf 
Über die Beerenfutichte einiger Caprifoliaceen.

\begin{tabular}{|c|c|c|c|c|c|c|}
\hline 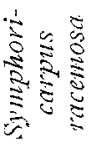 & & $\begin{array}{l}\infty \\
\dot{\varpi} \\
\ddot{=}\end{array}$ & 1 & & & 1 \\
\hline 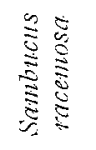 & $\begin{array}{l}\propto \\
\stackrel{\varrho}{\Xi}\end{array}$ & $\begin{array}{l}0 \\
\dot{0} \\
0 \\
0\end{array}$ & $\dot{0}$ & $\begin{array}{l}0 \\
\dot{0} \\
\bullet\end{array}$ & $\begin{array}{l}18 \\
0 \\
+1 \\
-1\end{array}$ & $\begin{array}{l}\ddot{\mathscr{D}} \\
\stackrel{\oplus}{0}\end{array}$ \\
\hline 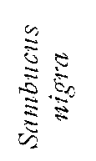 & $\dot{\stackrel{g}{g}}$ & $\stackrel{\infty}{0}$ & $\stackrel{8}{8}$ & $\dot{b}$ & $\begin{array}{l}10 \\
\infty 0 \\
+ \\
\stackrel{-}{-}\end{array}$ & $\begin{array}{l}\stackrel{\circ}{\rho} \\
\stackrel{\Xi}{\circ} \\
\dot{0}\end{array}$ \\
\hline 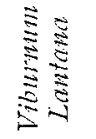 & $\begin{array}{l}\ddots \\
\stackrel{\Delta}{\theta}\end{array}$ & $\frac{9}{31}$ & 1 & $\ddot{0}$ & 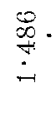 & I \\
\hline 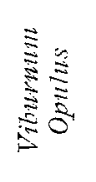 & $\begin{array}{l}\ddot{Q} \\
\dot{\Xi}\end{array}$ & $\begin{array}{l}\dot{\infty} \\
\stackrel{8}{\varrho}\end{array}$ & $\begin{array}{l}\ddot{Q} \\
\therefore\end{array}$ & $\bar{\alpha}$ & \begin{tabular}{l}
+1 \\
\multirow{2}{*}{} \\
$\dot{-}$ \\
$\rightarrow$
\end{tabular} & 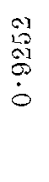 \\
\hline 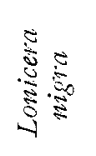 & $\begin{array}{l}0 \\
0 \\
0 \\
0 \\
0\end{array}$ & $\stackrel{\vec{H}}{\dot{b}}$ & $\frac{0}{\dot{0}}$ & $\begin{array}{l}0 \\
\infty\end{array}$ & $\underset{\stackrel{H}{g}}{\stackrel{H}{g}}$ & 1 \\
\hline 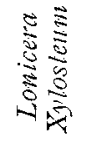 & $\begin{array}{l}0 \\
\infty \\
\dot{+} \\
\infty \\
\infty\end{array}$ & $\begin{array}{l}\infty \\
\dot{\theta} \\
\dot{\theta}\end{array}$ & $\begin{array}{l}\Phi \\
\dot{\forall}\end{array}$ & $\Leftrightarrow$ & 1 & 1 \\
\hline re & 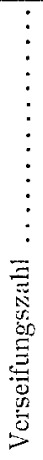 & 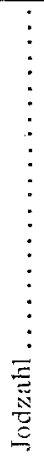 & 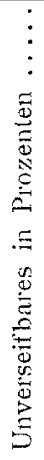 & 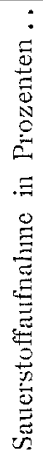 & 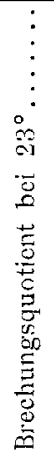 & 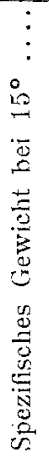 \\
\hline
\end{tabular}


310 G. Nowak und J. Zellner, Über die Beerenfrüchte einiger Caprifoliaceen.

Reinfett (Abrechnung der unverseifbaren Stoffe) deutlich hervor. Es handelt sich durchaus um trocknende und halbtrocknende Öle, eine Erscheinung, die auch bei anderen Beerensamenölen nicht selten beobachtet wird.

Schließlich sei auch erwähnt, daß über die Beeren zweier nahverwandter Arten, nämlich Viburnum nudum ${ }^{1}$ und Viburnum Lentago, ${ }^{2}$ einige, allerdings unvollständige Angaben vorliegen.

1 Chem. Zentralbl., 1909, I, 1660.

$\because$ Ebenda, 1911, I, 1753. 